\title{
Nanomedicine strategy for optimizing delivery to outer hair cells by surface-modified poly(lactic/ glycolic acid) nanoparticles with hydrophilic molecules
}

\author{
This article was published in the following Dove Press journal: \\ International Journal of Nanomedicine \\ 10 November 2016 \\ Number of times this article has been viewed
}

\section{Xingxing Wen, ${ }^{1,2}$ Shan \\ Ding, ',2 Hui Cai, ,,2 Junyi \\ Wang, ${ }^{3}$ Lu Wen, ' Fan Yang, ${ }^{1,2}$ Gang Chen ${ }^{1,2}$ \\ 'School of Pharmacy, ${ }^{2}$ Guangdong Provincial Key Laboratory of Advanced Drug Delivery, ${ }^{3}$ School of Public Health, Guangdong Pharmaceutical University, Guangzhou, People's Republic of China}

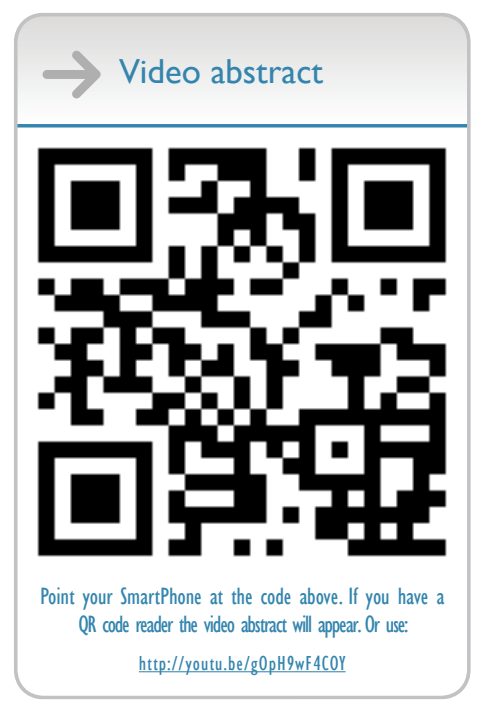

Correspondence: Lu Wen; Gang Chen School of Pharmacy, Guangdong Pharmaceutical University, Guangzhou 510006, People's Republic of China Tel +86 203935 2I 4I; +86203935 2117 Fax +86 2039352129

Email gywenl@I63.com; cg753@I26.com

\begin{abstract}
Targeted drug delivery to outer hair cells (OHCs) in the cochlea by nanomedicine strategies forms an effective therapeutic approach for treating hearing loss. Surface chemistry plays a deciding role in nanoparticle (NP) biodistribution, but its influence on such distribution in the cochlea remains largely unknown. Herein, we report the first systematic comparison of poly(lactic/glycolic acid) nanoparticles (PLGA NPs) with or without surface modification of hydrophilic molecules for optimizing the delivery to OHCs both in vitro and in vivo. NPs that were surface modified with poloxamer 407 (P407), chitosan, or methoxy poly(ethylene glycol) and the unmodified NPs were highly biocompatible with L929 and House Ear Institute-organ of Corti 1 cells as well as cochlear tissues. Interestingly, among all the examined NPs, P407-PLGA NPs showed the greatest cellular uptake and prominent fluorescence in cochlear imaging. More importantly, we provide novel evidence that the surface-modified NPs reached the organ of Corti and were transported into the $\mathrm{OHCs}$ at a higher level. Together, these observations suggest that surface modification with hydrophilic molecules will allow future clinical applications of PLGA NPs, especially P407-PLGA NPs, in efficient hearing loss therapy.
\end{abstract}

Keywords: inner ear drug delivery, PLGA nanoparticles, surface chemistry, cochlea, HEIOC1 cell

\section{Introduction}

According to the report from the World Health Organization, 5\% of the world's population (approximately 360 million people) suffers from a disabling hearing impairment. ${ }^{1}$ It is reported that noise exposure, ototoxic agents, aging, and some other factors result in the functional loss of outer hair cells (OHCs), bringing about hearing loss..$^{2-4}$ To improve the efficiency for treating hearing loss associated with $\mathrm{OHC}$ pathology, targeted drug delivery to OHCs in the organ of Corti (OC) of cochlea is required. ${ }^{5,6}$ Novel nanosized drug vehicles are thought to overcome the limitations of classic approaches through controlled or sustained release into the inner ear fluids, cellular cytoplasm, or the nucleus of targeted cells. ${ }^{7,8}$ Therefore, drugs have been delivered to the inner ear based on nanomedicine strategies using different types of nanocarriers. ${ }^{6,9}$ Among these nanocarrier materials, poly(lactic/glycolic acid) (PLGA), approved by the US Food and Drug Administration in multiple drug delivery systems in humans, has attracted considerable attention due to its high biodegradability and biocompatibility. ${ }^{10}$ More interestingly, it has been illustrated in our previous study that 
PLGA nanoparticles (NPs) loaded with single or compound drugs enhanced the local bioavailability of drugs following inner ear administration. ${ }^{11}$

Since the NP surface contacts with biological systems, their characteristics greatly impact NP performance in vivo and the overall effectiveness. ${ }^{5,12,13}$ Changing the surface chemistry of the engineered NPs with biocompatible hydrophilic polymers or copolymers increases the circulation time and delays the removal of NPs by the mononuclear phagocyte system, which prevents rapid clearance before reaching the targeted tissues and delivering drugs. ${ }^{12,14,15}$ Based on these background studies, we theorized that attachment of these hydrophilic molecules onto the NP surface would enhance nanocarrier delivery to $\mathrm{OHCs}$ in the cochlea. To verify this hypothesis, a deeper and comprehensive comparison of surface-modified PLGA NPs with various hydrophilic molecules for $\mathrm{OHC}$ delivery was performed.

We have recently developed a PLGA-based NP platform with different surface decorations by poloxamer 407 (P407), chitosan (CS), and methoxy poly(ethylene glycol) (mPEG), respectively. Herein, we systematically compare the NPs with and without surface modification as nanocarriers for delivery to OHCs both in vitro and in vivo. Our study highlighted that the efficiency for NP delivery to the inner ear and OHCs was improved by modifying the NP surface with hydrophilic molecules, especially with P407 decoration, as an optimizing strategy.

\section{Materials and methods Materials}

Coumarin-6 and polyvinyl alcohol 4-88 (PVA, molecular weight [MW] $31 \mathrm{kDa}$ ) were purchased from Sigma-Aldrich Co. (St Louis, MO, USA). 1,1'-Dioctadecyl-3,3,3',3tetramethylindotricarbocyanine iodide (DiR) was purchased from Molecular Probes (Thermo Fisher Scientific, Waltham, MA, USA). PLGA (LA/GA=75:25, MW $10 \mathrm{kDa}$ ) and mPEGPLGA (LA/GA =75:25, MW 10 kDa; mPEG, MW 1.9 kDa) were supplied by Shandong Medical Institute (Shandong, People's Republic of China). P407 (Pluronic F127) was a gift from BASF (Ludwigshafen, Germany) and used as supplied. CS (MW 300 kDa) was purchased from Zhejiang GoldenShell Pharmaceutical Co., Ltd (Zhejiang, People's Republic of China). Cell counting kit-8 (CCK-8) was purchased from Dojindo Molecular Technology Inc. (Shanghai, People's Republic of China). 4',6-Diamidino-2-phenylindole (DAPI) was purchased from Beyotime Institute of Biotechnology (Shanghai, People's Republic of China). All reagents were used as received without further purification.

\section{Preparation and size measurement of NPs}

PLGA NPs were prepared by the emulsion/solvent evaporation method. Briefly, $1 \mathrm{~mL}$ of dichloromethane-ethyl acetate (7:3) containing $30 \mathrm{mg}$ of PLGA and $60 \mu \mathrm{g}$ of coumarin-6 was added to $5 \mathrm{~mL}$ of $3 \%(\mathrm{w} / \mathrm{v})$ PVA aqueous solution on ice using a probe sonicator set at $190 \mathrm{~W}$ of energy output (Scientz Biotechnology Co. Ltd, Ningbo, People's Republic of China) for $100 \mathrm{~s}$ with pulses of $5 \mathrm{~s}$ on and $5 \mathrm{~s}$ off to form an oil-in-water emulsion. Then, this emulsion was diluted with a $0.5 \%(\mathrm{w} / \mathrm{v})$ PVA aqueous solution, followed by evaporation of organic solvents under magnetic stirring for $3 \mathrm{~h}$ to create coumarin-6 nanosuspension. CS-PLGA NPs were prepared by dissolving $30 \mathrm{mg}$ of PLGA and 60 $\mu \mathrm{g}$ of coumarin- 6 in $1 \mathrm{~mL}$ of ethyl acetate, and for P407PLGA NPs, PLGA and coumarin-6 were dissolved in 1 $\mathrm{mL}$ of dichloromethane, respectively. The organic phase was added to an aqueous solution consisting of $1 \%$ PVA (w/v) $-0.3 \%$ CS (w/v, CS was dissolved in $1 \%$ acetic acid solution) and 1\% PVA (w/v)-1\% P407 (w/v, P407 was dissolved in distilled water), respectively. For mPEG-PLGA NPs, $30 \mathrm{mg}$ of mPEG-PLGA and $60 \mu \mathrm{g}$ of coumarin-6 were dissolved in $1 \mathrm{~mL}$ of dichloromethane. Further steps performed were similar to those for PLGA NPs. DiR-loaded PLGA NPs were prepared with the same method as that for coumarin-6-loaded NPs, and the only difference was addition of $0.5 \mathrm{mg}$ of DiR in the organic solvent. The size of NPs was determined by a Delsa ${ }^{\mathrm{TM}}$ Nano $\mathrm{C}$ particle analyzer (Beckman Coulter Instruments, Brea, CA, USA).

\section{In vitro experiments}

\section{Cell culture}

The uptake and toxicity of NPs were examined in vitro using cells from a mouse fibroblast cell line L929 (Type Culture Collection of the Chinese Academy of Sciences, Shanghai, People's Republic of China), which were kept in modified Eagle medium ( $\alpha$-MEM; HyClone) supplemented with $10 \%$ fetal bovine serum, $100 \mathrm{IU} / \mathrm{mL}$ penicillin, and $100 \mathrm{IU} / \mathrm{mL}$ streptomycin at $37^{\circ} \mathrm{C}$ in $5 \% \mathrm{CO}_{2}$ using a humidified incubator (Thermo HERAcell 150i; Thermo Fisher Scientific).

Then, the House Ear Institute-organ of Corti 1 (HEI-OC1) cells, which were derived from the $\mathrm{OC}$ and obtained from the House Ear Institute (Los Angeles, CA, USA), were used to further test the cytotoxicity and cellular uptake of NPs. The cells were cultured in high-glucose Dulbecco's Modified Eagle's Medium (DMEM; Thermo Fisher Scientific) containing $10 \%$ fetal bovine serum at $33^{\circ} \mathrm{C}$ in $10 \% \mathrm{CO}_{2}$ using a humidified incubator (Thermo Forma 371, Thermo Fisher Scientific). 


\section{Cytotoxicity of NPs in $\mathrm{L} 929$ and HEI-OCI cells detected by CCK-8 assay}

Cell viability of NPs was determined using CCK-8 assay. L929 and HEI-OC1 cells were seeded at $1 \times 10^{4}$ cells per well onto 96-well plates and incubated for $24 \mathrm{~h}$, respectively. Blank NPs at different concentrations $(0.005,0.5,5,10,20$, 25 , and $40,60,80 \mathrm{mg} / \mathrm{mL}$ ) in diluted medium were added into both types of cells, respectively. After treatment for $24 \mathrm{~h}$, the NPs suspension was removed and the cells were rinsed twice with phosphate-buffered saline (PBS). Then, 10\% CCK-8 solution was added to each well and incubated for $2 \mathrm{~h}$. The absorbance was monitored at $450 \mathrm{~nm}$ in a microplate reader (RT-2100C; Rayto Life and Analytical Sciences Co. Ltd, Shenzhen, People's Republic of China). Cell viability was quantified by the ratio to the control, and the untreated cells were assigned a cell viability of $100 \%$.

\section{Observation of NP cellular uptake by confocal microscopy}

The cellular uptake and intracellular localization of NPs were confirmed by confocal microscopy. L929 and HEI-OC1 cells were seeded at $5 \times 10^{4}$ cells per well onto confocal special dishes and incubated for $24 \mathrm{~h}$, respectively. Then, the cells were incubated with coumarin-6-loaded NPs at a concentration of $40 \mu \mathrm{g} / \mathrm{mL}$ in serum-free medium for $1 \mathrm{~h}$. Afterward, the NPs were removed following a two-step washing with PBS, and then fixed with fresh $10 \%$ formalin for $10 \mathrm{~min}$ at room temperature. Next, these cells were incubated for $10 \mathrm{~min}$ with DAPI, a nuclei-specific dye with contrasting fluorescence, washed twice with PBS, and then analyzed under confocal microscope (LSM 710 Meta; Carl Zeiss Inc., Thornwood, NY, USA) with the following excitation and emission wavelengths: coumarin-6: excitation $453 \mathrm{~nm}$, emission band pass 504-530 $\mathrm{nm}$ and DAPI: excitation $405 \mathrm{~nm}$, emission 454-495 nm.

\section{Quantification of NP cellular uptake by flow cytometry}

The quantitative cellular uptake of NPs in L929 cells and HEI-OC1 cells was measured by flow cytometry. Briefly, the L929 and HEI-OC1 cells were seeded at $1 \times 10^{5}$ cells per well onto six-well plates and incubated for $24 \mathrm{~h}$, respectively. To determine the NPs uptake and loading extent, the cells were incubated with coumarin-6-loaded NPs at a concentration of $40 \mu \mathrm{g} / \mathrm{mL}$ in serum-free medium for $1 \mathrm{~h}$. Afterward, the cells were washed with PBS three times and harvested by trypsinization to obtain the suspension in PBS for monitoring. Green fluorescence-emitting coumarin-6 incorporated into different NPs served as a marker to quantitatively determine their cellular uptake by flow cytometer (BD Accuri C6; Becton Dickinson, Mississauga, ON, Canada), while $1 \times 10^{4}$ events were collected for each sample.

\section{In vivo experiments}

\section{Animals}

A total of 42 albino guinea pigs (250-300 g) of both sexes with normal Preyer reflexes were used in this study. They were obtained from the Laboratory Animal Center of Southern Medical University (Guangzhou, People's Republic of China). All animal studies were performed in accordance with the protocols approved by the Animal Ethical Committee (Guangdong Pharmaceutical University, Guangzhou, People's Republic of China); humane care was provided to all animals used in the studies. These animals had free access to food and water and were allowed a 1-week acclimation period before starting the experiment.

\section{NPs application to the inner ear}

The histocompatibility and distribution of four types of PLGA NPs in the inner ear were examined $24 \mathrm{~h}$ following intratympanic administration. Animals were anesthetized with ethyl carbamate $(1 \mathrm{~g} / \mathrm{kg})$ before the administration. Following each in vivo experiment, four types of NPs at a concentration of $25 \mathrm{mg} / \mathrm{mL}(0.1 \mathrm{~mL})$ were injected into the unilateral tympanic cavity of the guinea pigs $(n=3)$, respectively. Meanwhile, the control animals $(n=2)$ were treated with normal saline (NS).

\section{Detection of NPs in the cochlea by near-infrared fluorescence (NIRF) imaging system}

NPs in the cochlea were detected by an NIRF imaging system. After $24 \mathrm{~h}$ of treatment with DiR-loaded NPs or NS, the animals were decapitated under deep anesthesia, and bubbles were harvested rapidly and fixed with $10 \%$ formalin at $4^{\circ} \mathrm{C}$. Then, the signal of DiR in the NPs was evaluated by the NIRF imaging system (NIGHTOWL LB 983 NC100; Berthold Technologies, Bad Wildbad, Germany) with the excitation and emission wavelengths of 700 and $780 \mathrm{~nm}$, respectively. The statistical data obtained were expressed as the total amount of fluorescence and standard deviation.

\section{Distribution of NPs in the cochlear tissues and evaluation of the safety using paraffin sections}

Distribution of the NPs in cochlear tissues was examined on paraffin sections by confocal microscopy and their safety was evaluated through cochlear histopathology. Animals were sacrificed $24 \mathrm{~h}$ after administration of coumarin-6-loaded NPs 
or NS under deep anesthesia. After cardiac perfusion with NS and 10\% formalin, bubbles were harvested rapidly and fixed with the same fixative at $4^{\circ} \mathrm{C}$. The fixed inner ears were immersed in diluted nitric acid and stirred gently for approximately $48 \mathrm{~h}$. The decalcified specimens were dehydrated with ethanol solutions at gradient concentrations, cleared in xylene, and finally embedded in paraffin. Serial $4 \mu \mathrm{m}$ sections were cut from each paraffin block and mounted on glass slides. Then, the slides were used to observe the NPs in the cochlea under a fluorescence microscope (green channel) (Olympus Corporation, Tokyo, Japan), which were stained with DAPI to label the cell nucleus for observation in different cochlear tissues under confocal microscope (LSM 710 Meta, Carl Zeiss Inc.) or stained with hematoxylin and eosin to examine the histomorphology of the cochlear tissues in brightly fluorescent regions under the fluorescence microscope (Olympus Corporation).

\section{Location of NPs in OHCs by cochlear surface preparation}

Location of NPs in OHCs was visualized by cochlear surface preparation. As mentioned earlier, animals were sacrificed at intervals $24 \mathrm{~h}$ after the administration of NPs or NS; acoustic bullas were removed from them. Then, the bullas were rapidly opened and the fluid spaces were perfused with $10 \%$ formalin through the oval window and a fenestra in each semicircular canal. Membranous organs of the inner ear were then carefully dissected and fixed for $2 \mathrm{~h}$ at $4^{\circ} \mathrm{C}$ under high magnification. After washing in PBS, the fixed tissues were stained with DAPI for $10 \mathrm{~min}$ and washed twice with PBS. After staining, the basilar membrane with OC was placed on glass slides in glycerin and coverslipped. Then the coverslips were sealed with nail polish to protect the specimens from desiccation and the specimens were examined by confocal microscope (LSM 510 Meta, Carl Zeiss Inc.).

\section{Statistical analysis}

The fluorescence intensity was determined by AxioVision Rel. 4.7 (Carl Zeiss Inc.), and the average fluorescence value in each frame was computed using region of interest analysis. Herein, regions of interest are the areas corresponding to each cell or cochlear tissue. Analysis of variance followed by multiple comparisons test was used in Statistical Package for the Social Sciences (SPSS) 19.0 (IBM Corporation, Armonk, NY, USA) to determine significant differences between groups, and $P$-values less than 0.05 were considered statistically significant. The half-maximal inhibitory concentration (IC50 value) was obtained using SPSS software 19.0.
Semi-quantitative fluorescent analysis was used to compare the uptake or biodistribution of different surface-modified PLGA NPs, and F-value was introduced to represent the ratio of the uptake or biodistribution of various PLGA NPs to that of unmodified PLGA NPs.

$$
F \text {-value }=\frac{\mathrm{F}_{\mathrm{x}}-\mathrm{B}}{\mathrm{F}_{0}-\mathrm{B}},
$$

where $\mathrm{F}_{\mathrm{x}}$ is the mean fluorescence intensity (MFI) of different types of PLGA NPs, $F_{0}$ the MFI of unmodified PLGA NPs, and $\mathrm{B}$ is the MFI of control background.

\section{Results and discussion}

The application of nanomedicine in hearing loss therapy is primarily driven by the need for increasing the therapeutic index of a given drug, which may be achieved by optimizing the nanocarriers based on surface modification. Various hydrophilic molecules (P407, CS, and mPEG) were chosen to decorate PLGA NPs. Since it has been proven that nanocarriers transport across the middle-inner ear barriers in a size-dependent manner, ${ }^{16}$ we prepared various surfacemodified NPs using different solvent systems to obtain NPs with similar sizes. ${ }^{17}$ In our study, we formulated four types of PLGA-based NPs with or without surface modification, that is, PLGA NPs, P407-PLGA NPs, CS-PLGA NPs, and mPEG-PLGA NPs, respectively (Figure S1). CS coating was achieved by electrostatic interaction between negatively charged PLGA chains and positively charged CS. ${ }^{18}$ P407 copolymers, containing polyethylene oxide (hydrophilic) and polypropylene oxide (hydrophobic) moieties, were adsorbed to particle surfaces from an aqueous solution through hydrophobic interaction of the hydrophobic moiety (the anchor block) with the particle surface, ${ }^{19}$ and mPEG-PLGA polymer was used to prepare mPEG-modified PLGA-NPs. These NPs possessed similar tailored average hydrodynamic sizes (100-200 nm) and relatively uniform size distribution (Table S1; Figures S2 and S3).

We first investigated the cytotoxicity and the uptake of NPs in L929 cells after treatment with various NPs, as depending on the modified molecules, the cytotoxicity and the uptake profiles can change. ${ }^{20}$ L929, a normal cell line, has been widely used in a series of studies including drug delivery to the inner ear. ${ }^{21,22}$ As shown in Figure 1A, the viability of L929 cells remained high after being treated with the four types of NPs at various concentrations, even up to $25 \mathrm{mg} / \mathrm{mL}$ (the original NPs suspension), suggesting that PLGA-based 


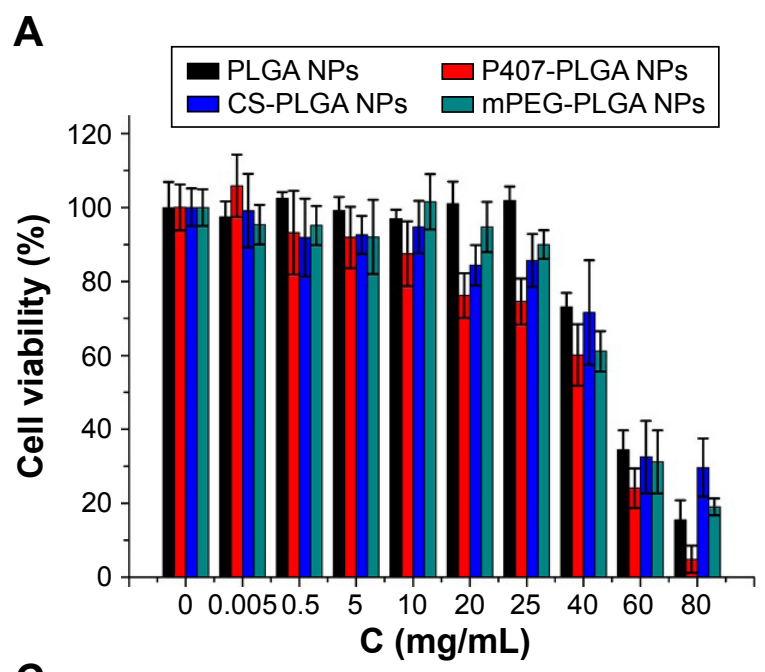

C

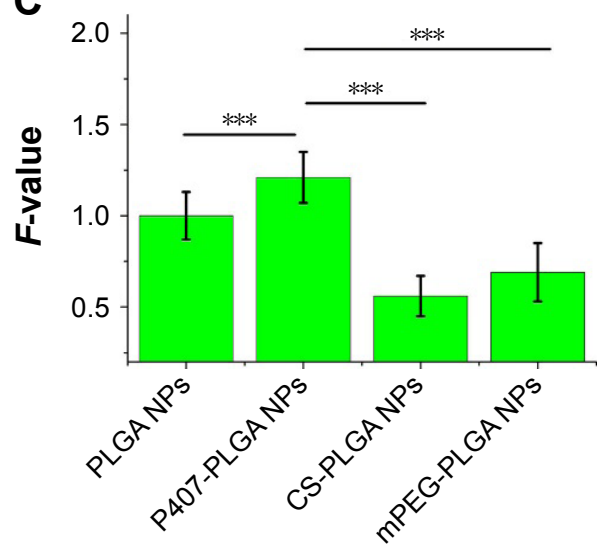

B
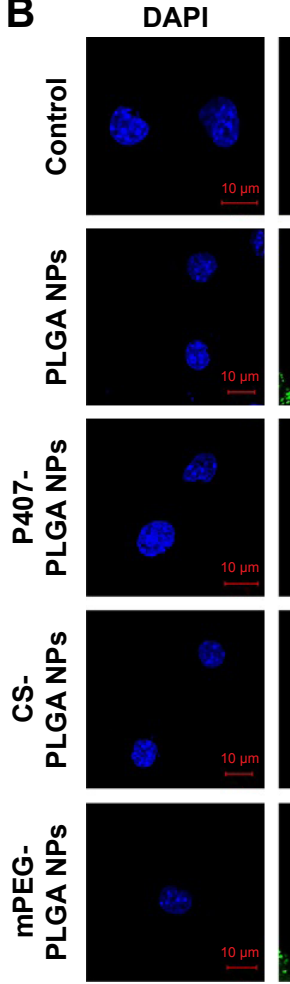

Merged
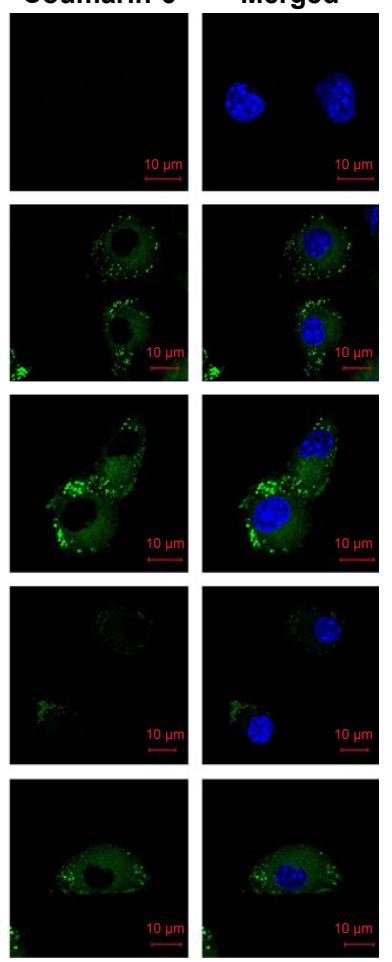

D
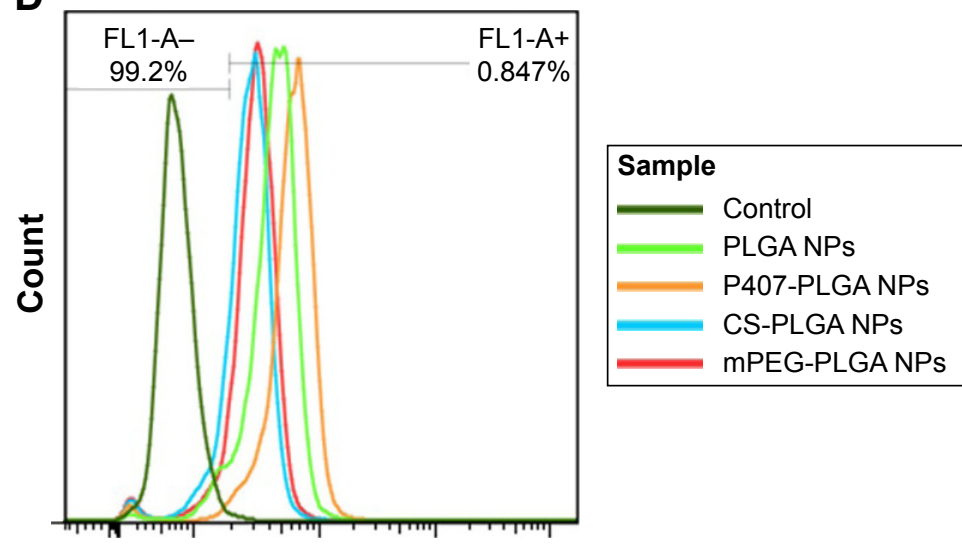

Figure I Cytotoxicity and cellular uptake of different surface-modified PLGA NPs in L929 cells.

Notes: (A) Cytotoxicity of NPs after incubation for $24 \mathrm{~h}$ as determined by CCK-8 assay. Percent viability of cells was expressed relative to the control cells. Results are shown as mean $\pm S D(n=6)$. (B, C) Confocal fluorescent microscopy and semi-quantitative fluorescence analysis showing the cellular uptake of different surface-modified, coumarin-6-loaded NPs after coincubation for I h $(* * * P<0.00 \mathrm{I})$. Scales in the figure represent I0 $\mu \mathrm{m}$. F-value is the ratio of MFI in each cell of various PLGA NPs to that of unmodified PLGA NPs. Results are shown as mean \pm SD $(n=30)$. (D) Cellular uptake of surface-modified coumarin-6-loaded NPs after coincubation for I h detected by flow cytometry.

Abbreviations: CCK-8, cell counting kit-8; CS, chitosan; DAPI, 4',6-diamidino-2-phenylindole; MFI, mean fluorescence intensity; mPEG, methoxy poly(ethylene glycol); NPs, nanoparticles; P407, poloxamer 407; PLGA, poly(lactic/glycolic acid); SD, standard deviation; h, hour.

nanocarriers were biocompatible with normal cells. The IC50 values of unmodified, P407-, CS-, and mPEG-PLGA NPs were $53.56 \pm 1.03,41.51 \pm 2.41,56.06 \pm 6.36$, and $51.03 \pm 2.43 \mathrm{mg} / \mathrm{mL}$, respectively $(\mathrm{n}=6)$. Then, the cellular uptake and intracellular localization of coumarin-6-loaded NPs into L929 cells were observed by confocal microscopy. Figure 1B shows the colocalization of NPs (green) and DAPI-stained cell nucleus (blue) in L929 cells, suggesting 
PLGA-based NPs were internalized with a spotty distribution around the nucleus. Clearly, surface modification was a governing factor for the uptake of PLGA-based NPs by L929 cells, as indicated by the fluorescence intensity of internalized NPs. Semi-quantitative fluorescence analysis revealed that P407-PLGA NPs had the greatest cellular uptake with significantly higher $F$-value than those of other kinds of NPs (all $P<0.001$ ), followed by the unmodified, mPEG-, and CS-PLGA NPs, successively (Figures $1 \mathrm{C}$ and S4). The uptake efficiencies of unmodified, P407-, CS-, and mPEG-PLGA NPs in L929 cells were further quantified by flow cytometry and were found to be $95.3 \%, 98.7 \%, 76.2 \%$, and $87.8 \%$, respectively (Figures 1D and $\underline{\mathrm{S} 5}$ ). Therefore, all the PLGAbased NPs, especially P407-PLGA NPs, had considerable uptake in L929 cells.

HEI-OC1, another cell model chosen in our study, is a conditionally immortalized mouse cell line derived from the postnatal OC displaying various phenotypes for nonsensory cells and used as a potential model system to screen otological drugs. ${ }^{23}$ We then investigated the viability of HEI-OC1 cells and the uptake of NPs in them after treatment with PLGA-based NPs. All PLGA-based NPs, and even the original NP suspension, were nontoxic to HEI-OC1 cells after coincubation for $24 \mathrm{~h}$ (Figure 2A). Taking the results of L929 cells into consideration simultaneously, $25 \mathrm{mg} / \mathrm{mL}$ NPs suspension was safe and appropriate for cochlear application in the following animal experiments to ensure their visualization in vivo. The IC50 values of unmodified, P407-,

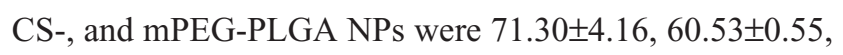
$65.39 \pm 0.47$, and $81.70 \pm 1.04 \mathrm{mg} / \mathrm{mL}$, respectively $(\mathrm{n}=6)$. Identical to the cytotoxicity against L929 cells, P407-PLGA NPs had the highest IC50 value, which may be explained by the higher uptake amount of NPs than those of others. The uptake of different NPs by HEI-OC1 cells was observed by confocal microscopy and quantified by flow cytometry. Similar to the intracellular localization and uptake of NPs in L929 cells, PLGA-based NPs were uniformly distributed throughout the cytoplasm and P407-PLGA NPs had the greatest cellular uptake, which was 1.33-, 1.46-, and 3.14-fold those of unmodified, CS-, and mPEG-PLGA NPs, respectively (all $P<0.01$ ) (Figures 2B, C, and $\underline{\mathrm{S} 6}$ ). The uptake efficiencies of unmodified, P407-, CS-, and mPEG-PLGA NPs were $79.7 \%, 91.4 \%, 58.3 \%$, and $48.1 \%$, respectively (Figures 2D and S7). Remarkably, quantification of the flow cytometric analysis revealed that uptake in both cells was increased by applying P407 for surface decoration. Changing the physicochemical parameters of NP surface by coating P407 may facilitate this uptake process, during which the interaction between poloxamer and the cell membrane promotes endocytosis of NPs. ${ }^{24,25}$ It has been confirmed that glycerol monooleate-based NPs with high positive surface charge were more prone for uptake by L929 cells, ${ }^{21}$ but the uptake of CS-PLGA NPs with positive surface charge was less than that of unmodified PLGA NPs in our study. This difference may result from the surface charge density of NPs. At the positive surface charges of $20 \%-40 \%$, NPs remain on the bilayer surface instead of being taken up by cells. ${ }^{26,27}$ For mPEG-PLGA NPs, significant increase in surface hydrophilicity may decrease the uptake efficiency compared to that of unmodified NPs. It is well documented that hydrophilic NPs tended to reside on the membrane surface, ${ }^{28,29}$ whereas hydroxyl-functionalized fullerenes were adsorbed on the bilayer surface because the cellular uptake rate was reduced by nine orders of magnitude..$^{30}$

We also assessed whether different NPs would induce histological changes in the cochlea through intratympanic administration, as effectively delivering therapeutic agents to the inner ear without damaging the integrity of delicate structures is most challenging. ${ }^{31}$ Light microscopy with hematoxylin and eosin-stained paraffin sections did not reveal any inflammation in the inner ear. Individual cochlear tissues, including stria vascularis, spiral ligament, OC, and spiral ganglion cells, barely underwent morphological alterations after application of NPs for $24 \mathrm{~h}$ (Figure S8). This was consistent with the observation of cytotoxicity against L929 and HEI-OC1 cells.

Next, we encapsulated a near-infrared dye DiR into the NPs to study their cochlear entry by using an NIRF imaging system, owing to the extremely low tissue autofluorescence and high tissue penetration depth in the NIR spectrum window. ${ }^{32}$ By $24 \mathrm{~h}$ postinjection of P407-PLGA NPs, stronger fluorescence signal emitted from the excised cochleae than from those applied other NPs (Figure 3A). Moreover, as shown in Figure 3B, the excised cochleae treated with modified NPs showed higher fluorescence intensity than those treated with unmodified NPs. Particularly, the fluorescence intensity of P407-PLGA-NP-treated cochleae was 2.4-, 2.0-, and 1.55-fold those of the cochleae treated with unmodified, CS-, and mPEG-PLGA NPs, respectively (all $P<0.05$ ). Therefore, hydrophilic coating of PLGA NPs, especially P407 modification, played a key role in inner ear transport.

We then investigated NP distribution in the whole cochlear tissue and the individual cochlear structures by fluorescence microscopy or confocal microscopy, since the impact of surface modification of NPs with hydrophilic groups on the distribution of NP in the cochlear tissue has 

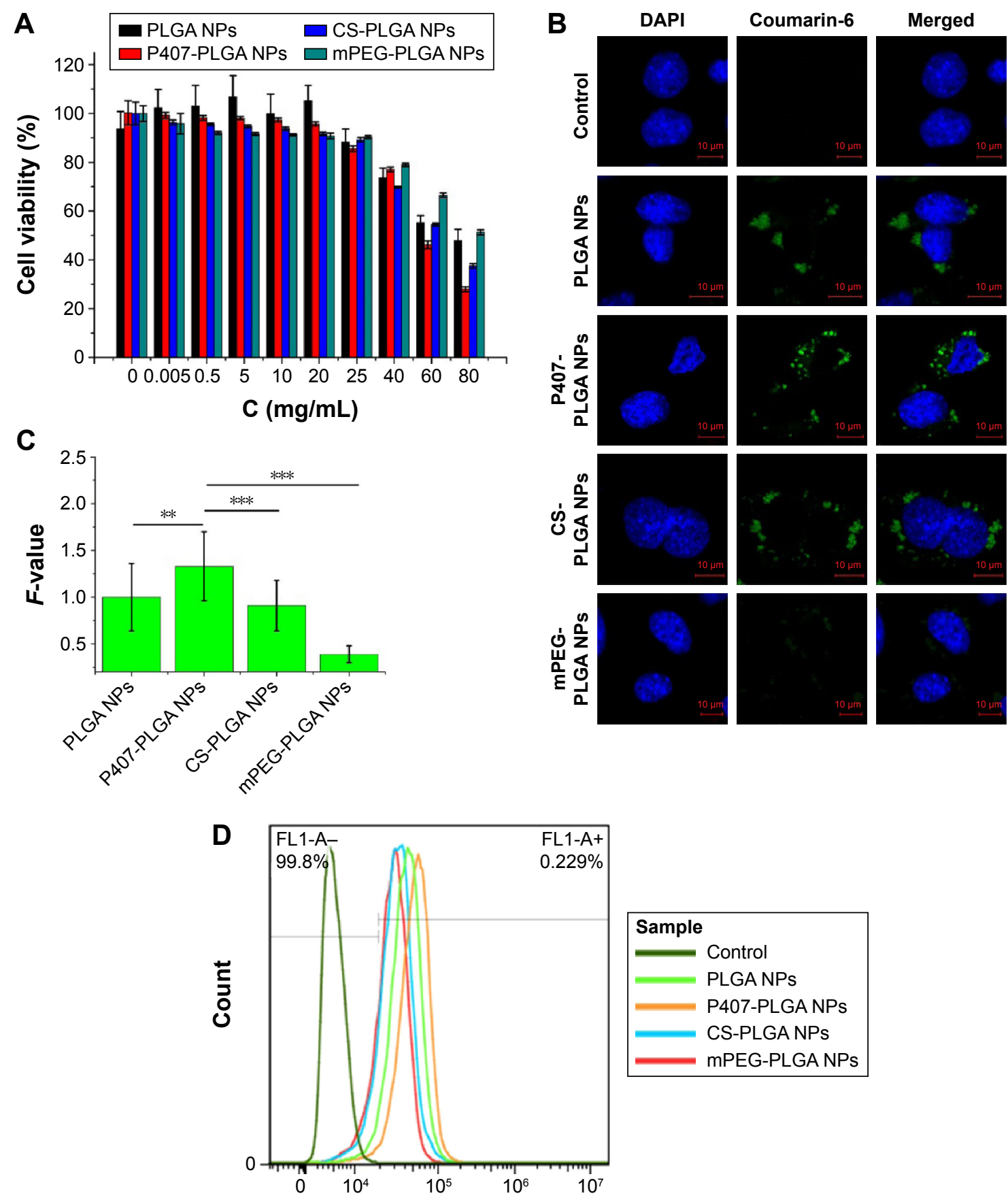

Figure 2 Cytotoxicity and cellular uptake of different surface-modified PLGA NPs in HEI-OCI cells.

Notes: (A) Cytotoxicity of NPs after incubation for $24 \mathrm{~h}$ as determined by CCK-8 assay. Percent viability of cells was expressed relative to the control cells. Results are shown as mean $\pm S D(n=6)$. (B, C) Confocal fluorescent microscopy and semi-quantitative fluorescence analysis showing the cellular uptake of different surface-modified, coumarin-6-loaded NPs after coincubation for I h $(* * P<0.01$, $* * * P<0.00 \mathrm{I})$. Scales in the figure represent $10 \mu \mathrm{m}$. F-value is the ratio of MFI in each cell of various PLGA NPs to that of unmodified PLGA NPs. Results are shown as mean \pm SD $(n=30)$. (D) Cellular uptake of surface-modified, coumarin-6-loaded NPs after coincubation for I $h$ detected by flow cytometry.

Abbreviations: CCK-8, cell counting kit-8; CS, chitosan; DAPI, 4',6-diamidino-2-phenylindole; MFI, mean fluorescence intensity; mPEG, methoxy poly(ethylene glycol); NPs, nanoparticles; P407, poloxamer 407; PLGA, poly(lactic/glycolic acid); SD, standard deviation; h, hours.

not been evaluated until now. In the paraffin sections of the cochlear tissue, more prominent fluorescence signal was observed from the apex and basal turns of the cochlea for surface-modified NPs compared with those of unmodified PLGA NPs (Figure 4). The signal of P407-PLGA NPs was significantly stronger (1.44-fold) than that of unmodified NPs, as evidenced by the semi-quantitative fluorescence analysis $(P<0.05)$ (Figure 4G). Besides, surface-modified PLGA NPs were considerably localized in spiral ligament, stria vascularis, OC, and spiral ganglion cells, whereas unmodified 
A

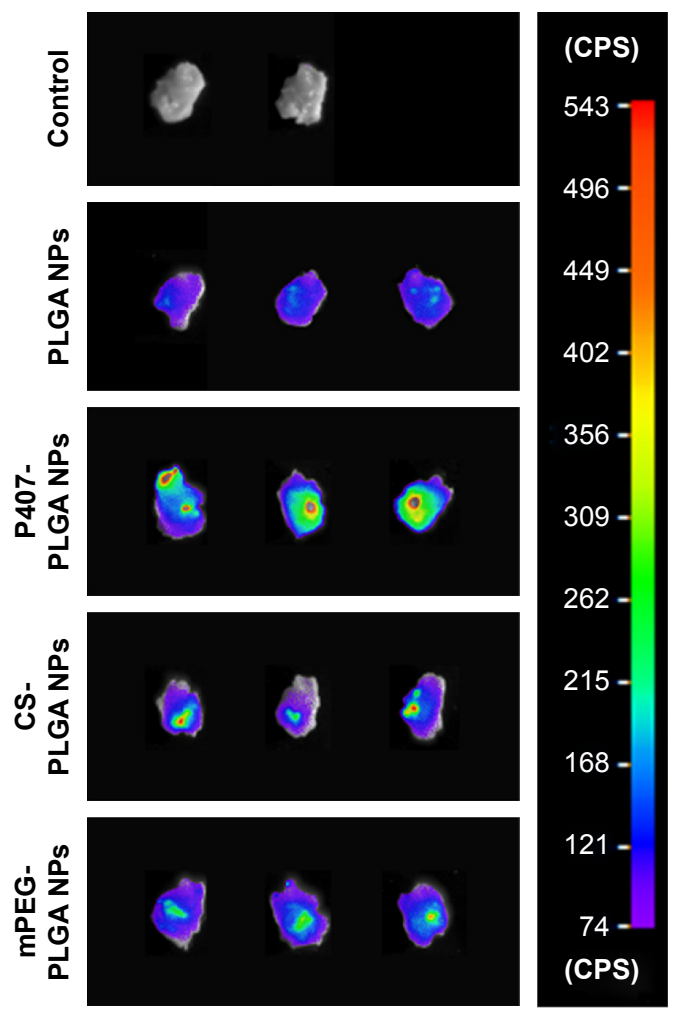

B

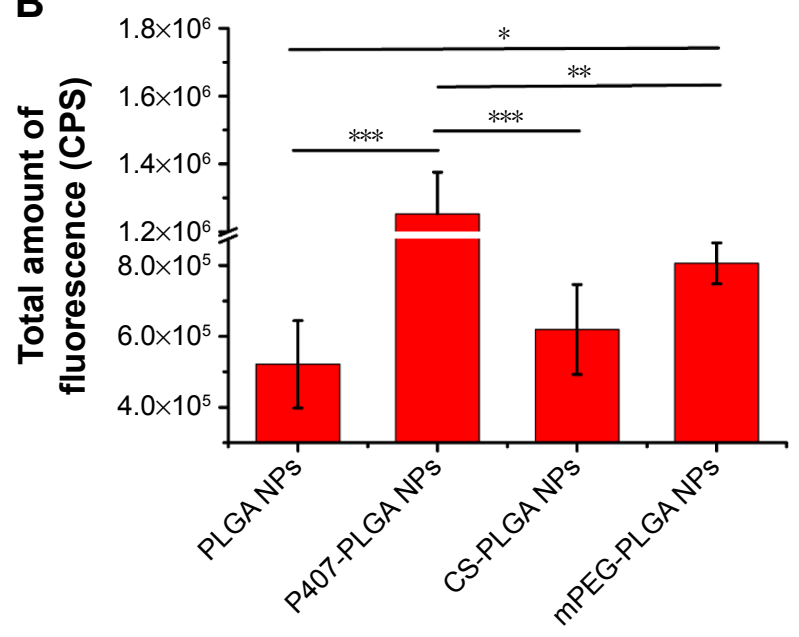

Figure 3 Cochlear entry of different surface-modified DiR-loaded NPs 24 h after intratympanic administration.

Notes: (A) Fluorescence images of excised cochleae $24 \mathrm{~h}$ after intratympanic administration of various surface-modified DiR-loaded NPs. (B) Fluorescence quantification of the total amount of fluorescence in the excised cochleae $\left({ }^{*} P<0.05\right.$, $* * P<0.0$ I, $* * * P<0.00 I)$. Results are shown as mean $\pm S D(n=3)$.

Abbreviations: CS, chitosan; DiR, I,I'-dioctadecyl-3,3,3',3-tetramethylindotricarbocyanine iodide; mPEG, methoxy poly(ethylene glycol); NPs, nanoparticles; P407, poloxamer 407; PLGA, poly(lactic/glycolic acid); SD, standard deviation; $h$, hours.

PLGA NPs were marginally distributed (Figure S9). Moreover, PLGA NPs with P407 modification accumulated to the greatest extent in the cochlear tissues (Figure 4H). This was in line with the result of NIRF imaging for the excised cochleae that engineered NP surfaces with hydrophilic molecules promoted the entry of NPs into the cochlea and their approach to the cochlear tissues. Particularly, P407 modification had a notable effect on NP distribution in vivo.

Finally, we monitored the entry of different NPs to OHCs in vivo by cochlear surface preparation (Figure 5). Stronger fluorescence signal around the $\mathrm{OHC}$ nucleus was found in animals given surface-modified NPs than that in unmodified NP-injected ones. Moreover, the fluorescence signals of P407-PLGA and mPEG-PLGA NPs were obviously detected in OHCs along the full length of the cochlea, whereas that CS-PLGA NPs were weaker. Semi-quantitative fluorescence analysis suggested that P407-PLGA NPs accumulated extensively and were located more homogeneously in OHCs. Meanwhile, the amount of fluorescence of P407-PLGA NPs taken up by the OHCs was 1.95-, 1.76-, and 1.39-fold those of unmodified, CS-, and mPEG-PLGA NPs, respectively (all $P<0.001$ ). Accordingly, coating PLGA NPs with hydrophilic molecules, especially P407, facilitated their transport into the OHCs. In general, in order to achieve cellular targeting in the cochlea, ligands known to bind the surface receptors of selected cells should be conjugated to engineered NPs. For example, polymersome surface functionalized with $\mathrm{hNgfEE}$ peptide (a nerve growth factor-derived ligand) shows great potential for specific targeting to spiral ganglion neurons, ${ }^{33}$ while engineered the polymersomes with prestin-binding peptides can promote their targeting to the OHCs. ${ }^{5}$ In our study, the PLGA-based NPs modified with hydrophilic molecules delivered their cargo to the OHCs more effectively than unmodified NPs did, indicating that hydrophilic molecules could also play the role of ligands for entry to $\mathrm{OHC}$.

The results of our study confirmed the hypothesis that surface modification with hydrophilic molecules promoted NP distribution in the cochlea and the OHCs. Surface attachments, P407, CS, and mPEG, enhanced cochlear entry in vivo, which may be attributed to the change in NP surface chemistry that prevents the clearance by ciliated epithelia in the tympanic cavity by blocking the hydrophobic interactions. ${ }^{14,34}$ Furthermore, by interacting with the cell membranes, $\mathrm{P} 407$ decreased the microviscosity and formed pores on the membrane, ${ }^{35-38}$ which may result in higher cellular uptake in the OHCs. Notably, in contrast to the little cellular uptake in vitro, mPEG-PLGA NPs were well distributed in vivo, probably due to their highest accumulation in the tissue among all kinds of NPs. ${ }^{39}$ Similarly, despite less cellular uptake in vitro, more CS-PLGA NPs reached OHCs than PLGA NPs did. Possibly, the NPs passed through 

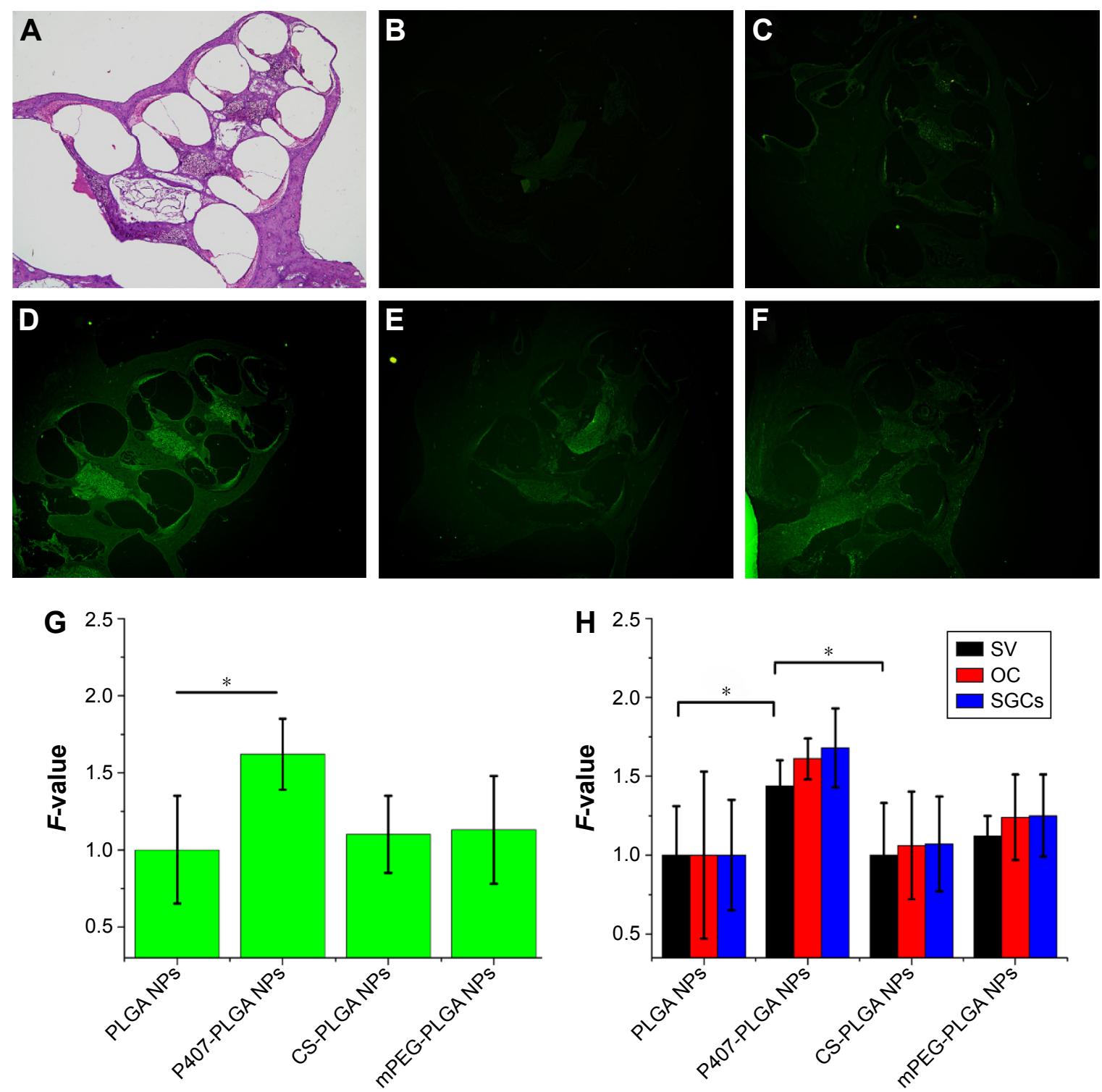

Figure 4 Distribution of different surface-modified, coumarin-6-loaded NPs in the cochlea $24 \mathrm{~h}$ after intratympanic application.

Notes: Images were taken with the same magnification (I0×4). (A) HE-stained paraffin sections of the cochlea axis. (B) Control, NS. (C) PLGA NPs. (D) P407-PLGA NPs. (E) CS-PLGA NPs. (F) mPEG-PLGA NPs. (G) Fluorescence quantification of cochleae $(* P<0.05)$. (H) Fluorescence quantification of cochlear tissues $(* P<0.05)$. $F$-value is the ratio of MFI in the cochlea or different cochlear tissues of various PLGA NPs to that of unmodified PLGA NPs. Results are shown as mean \pm SD ( $n=3$ ).

Abbreviations: CS, chitosan; HE, hematoxylin and eosin; MFI, mean fluorescence intensity; mPEG, methoxy poly(ethylene glycol); NPs, nanoparticles; NS, normal saline; OC, organ of Corti; P407, poloxamer 407; PLGA, poly(lactic/glycolic acid); SD, standard deviation; SGCs, spiral ganglion cells; SV, stria vascularis; h, hours.

the tight epithelial layer by a transcellular pathway in vivo and then through the loosely arranged connective tissue layer and the endothelial layer by an intercellular pathway. ${ }^{21,40}$

\section{Conclusion}

In summary, we prepared a series of surface-modified PLGA NPs using different hydrophilic molecules and investigated their transport into OHCs in vitro and in vivo, as well as compared them with unmodified NPs. The cytotoxicity, cellular uptake, biocompatibility, cochlear distribution, and location of these NPs were ascertained, which suggested that the PLGA-based nanocarriers were biocompatible and the hydrophilic coating had a notable effect on NP distribution. It was confirmed that the optimizing design using surfacemodified PLGA NPs with hydrophilic molecules, especially for PLGA NPs functionalized with P407, had greater capability to facilitate their entry into OHCs, indicating that the hydrophilic molecules could also play the role of ligands for OHC entry. As a result, nanomedicine strategy employing hydrophilic molecule-coated PLGA NPs will broaden the 

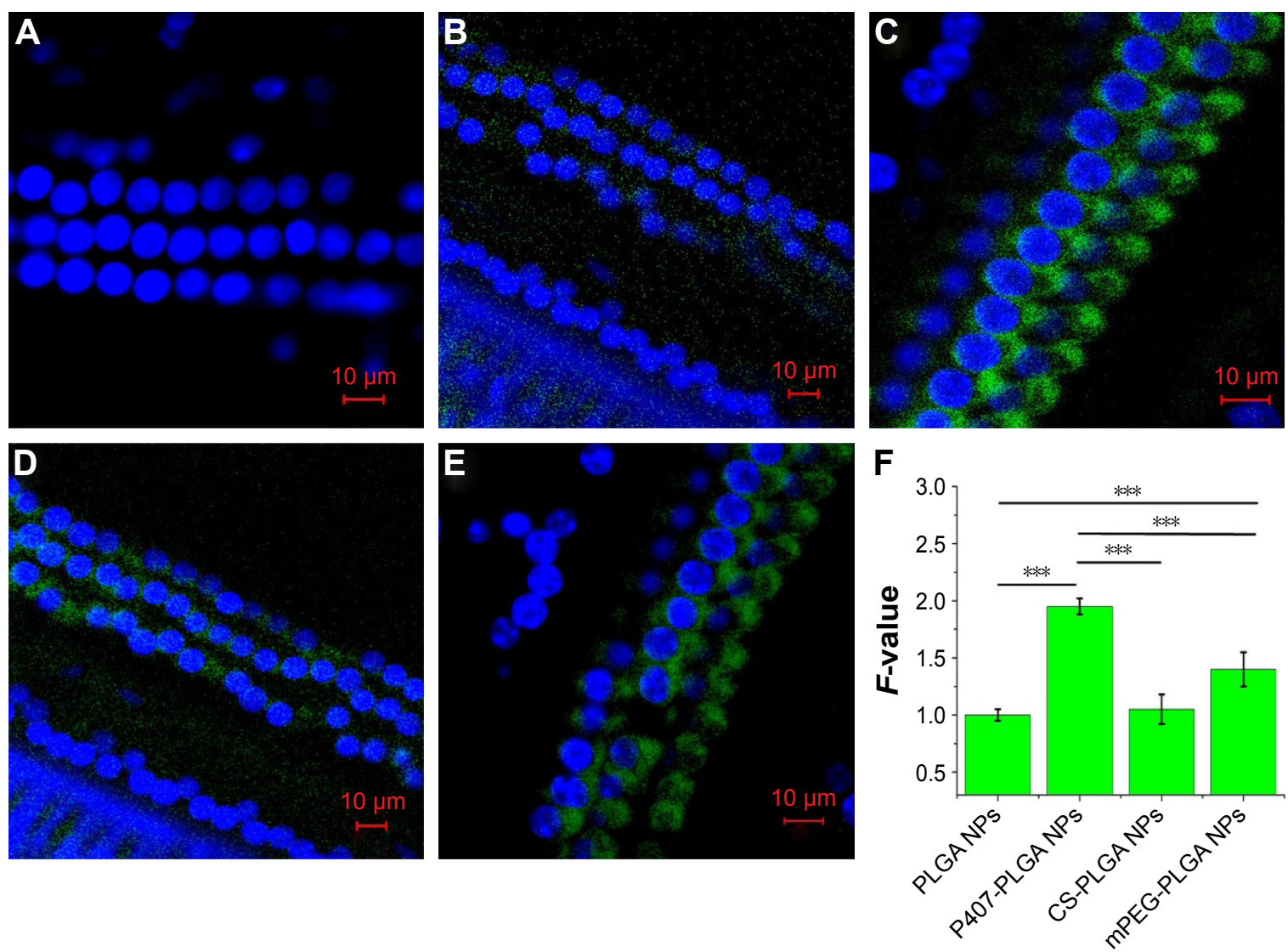

Figure 5 Localization of different surface-modified, coumarin-6-loaded NPs in OHCs 24 h after intratympanic application.

Notes: Scales in the figure represent $10 \mu \mathrm{m}$. (A) Control, NS. (B) PLGA NPs. (C) P407-PLGA NPs. (D) CS-PLGA NPs. (E) mPEG-PLGA NPs. (F) Fluorescence quantification of OHCs (***P<0.00I). F-value is the ratio of MFI in each OHC of various PLGA NPs to that of unmodified PLGA NPs. Results are shown as mean \pm SD ( $\mathrm{n}=10$ ).

Abbreviations: CS, chitosan; MFI, mean fluorescence intensity; mPEG, methoxy poly(ethylene glycol); NS, normal saline; NPs, nanoparticles; OHCs, outer hair cells; P407, poloxamer 407; PLGA, poly(lactic/glycolic acid); SD, standard deviation; h, hours.

way for a more efficient and more specific treatment for hearing loss originating from the impairment of OHCs.

\section{Acknowledgments}

This work was supported by the National Nature Science Foundation of China (Grant No 81274097), the Pearl River S\&T Nova Program of Guangzhou (Grant No 2013J2200059), and the Cultivation Foundation for Distinguished Young Teachers in Higher Education of Guangdong (Grant No Yq2013099).

\section{Disclosure}

The authors report no conflicts of interest in this work.

\section{References}

1. Moser T. Gene therapy for deafness: how close are we? Sci Transl Med. 2015;7(295):295fs 28

2. Han WJ, Shi XR, Nuttall A. Noise-induced nitrotyrosine increase and outer hair cell death in guinea pig cochlea. Chin Med J (Engl). 2013;126(15):2923-2927.

3. Ding D, Jiang H, Salvi RJ. Mechanisms of rapid sensory hair-cell death following coadministration of gentamicin and ethacrynic acid. Hear Res. 2010;259(1-2):16-23.
4. Altschuler RA, Dolan DF, Halsey K, et al. Age-related changes in auditory nerve-inner hair cell connections, hair cell numbers, auditory brain stem response and gap detection in UM-HET4 mice. Neuroscience. 2015;292:22-33.

5. Surovtseva EV, Johnston AH, Zhang W, et al. Prestin binding peptides as ligands for targeted polymersome mediated drug delivery to outer hair cells in the inner ear. Int J Pharm. 2012;424(1-2):121-127.

6. Li L, Chao T, Brant J, O’Malley B Jr, Tsourkas A, Li D. Advances in nano-based inner ear delivery systems for the treatment of sensorineural hearing loss. Adv Drug Deliv Rev. Epub 2016 Jan 12.

7. Poe DS, Pyykkö I. Nanotechnology and the treatment of inner ear diseases. Wiley Interdiscip Rev Nanomed Nanobiotechnol. 2011;3(2): 212-221.

8. Martín-Saldaña S, Palao-Suay R, Trinidad A, Aguilar MR, Ramírez-Camacho R, San Román J. Otoprotective properties of $6 \alpha$-methylprednisolone-loaded nanoparticles against cisplatin: in vitro and in vivo correlation. Nanomedicine. 2016;12(4):965-976.

9. Chen G, Zhang X, Yang F, Mu L. Disposition of nanoparticle-based delivery system via inner ear administration. Curr Drug Metab. 2010; 11(10):886-897.

10. Danhier F, Ansorena E, Silva JM, Coco R, Le Breton A, Pr at V. PLGAbased nanoparticles: an overview of biomedical applications. J Control Release. 2012;161(2):505-522.

11. Cai H, Wen X, Wen L, et al. Enhanced local bioavailability of single or compound drugs delivery to the inner ear through application of PLGA nanoparticles via round window administration. Int $J$ Nanomedicine. 2014;9:5591-5601. 
12. Amin ML, Joo JY, Yi DK, An SS. Surface modification and local orientations of surface molecules in nanotherapeutics. J Control Release. 2015;207:131-142.

13. Wang $\mathrm{H}$, Zhao $\mathrm{Y}$, Wang $\mathrm{H}$, et al. Low-molecular-weight protaminemodified PLGA nanoparticles for overcoming drug-resistant breast cancer. J Control Release. 2014;192:47-56.

14. Esmaeili F, Ghahremani MH, Esmaeili B, Khoshayand MR, Atyabi F, Dinarvand R. PLGA nanoparticles of different surface properties: preparation and evaluation of their body distribution. Int J Pharm. 2008; 349(1-2):249-255.

15. Mistry A, Stolnik S, Illum L. Nose-to-brain delivery: investigation of the transport of nanoparticles with different surface characteristics and sizes in excised porcine olfactory epithelium. Mol Pharm. 2015; 12(8):2755-2766.

16. Zou J, Sood R, Ranjan S, et al. Size-dependent passage of liposome nanocarriers with preserved posttransport integrity across the middleinner ear barriers in rats. Otol Neurotol. 2012;33(4):666-673.

17. Sahana DK, Mittal G, Bhardwaj V, Kumar MN. PLGA nanoparticles for oral delivery of hydrophobic drugs: influence of organic solvent on nanoparticle formation and release behavior in vitro and in vivo using estradiol as a model drug. J Pharm Sci. 2008;97(4):1530-1542.

18. Tahara K, Samura S, Tsuji K, et al. Oral nuclear factor-kB decoy oligonucleotides delivery system with chitosan modified poly(D,Llactide-co-glycolide) nanospheres for inflammatory bowel disease. Biomaterials. 2011;32(3):870-878.

19. Redhead HM, Davis SS, Illum L. Drug delivery in poly(lactide-coglycolide) nanoparticles surface modified with poloxamer 407 and poloxamine 908: in vitro characterrisation and in vivo evaluation. J Control Release. 2001;70(3):353-363.

20. Grabowski N, Hillaireau H, Verqnaud J, et al. Toxicity of surfacemodified PLGA nanoparticles toward lung alveolar epithelial cells. Int J Pharm. 2013;454(2):686-694.

21. Liu H, Chen S, Zhou Y, et al. The effect of surface charge of glycerol monooleate-based nanoparticles on the round window membrane permeability and cochlear distribution. J Drug Target. 2013;21(9): 846-854.

22. Zhang W, Zhang Y, Löbler M, et al. Nuclear entry of hyperbranched polylysine nanoparticles into cochlear cells. Int J Nanomedicine. 2011;6:535-546.

23. Kalinec GM, Webster P, Lim DJ, Kalinec F. A cochlear cell line as an in vitro system for drug ototoxicity screening. Audiol Neurootol. 2003; 8(4):177-189.

24. Chang J, Jallouli Y, Kroubi M, et al. Characterization of endocytosis of transferrin-coated PLGA nanoparticles by the blood-brain barrier. Int J Pharm. 2009;379(2):285-292.

25. Petersen S, Steiniger F, Fischer D, Fahr A, Bunjes H. The physical state of lipid nanoparticles influences their effect on in vitro cell viability. Eur J Pharm Biopharm. 2011;79(1):150-161.
26. Beddoes CM, Case CP, Briscoe WH. Understanding nanoparticle cellular entry: a physicochemical perspective. Adv Colloid Interface Sci. 2015;218:48-68.

27. da Rocha EL, Caramori GF, Rambo CR. Nanoparticle translocation through a lipid bilayer tuned by surface chemistry. Phys Chem Chem Phys. 2013;15(7):2282-2290.

28. Spurlin TA, Gewirth AA. Effect of C60 on solid supported lipid bilayers. Nano Lett. 2007;7(2):531-535.

29. Li Y, Chen X, Gu N. Computational investigation of interaction between nanoparticles and membranes: hydrophobic/hydrophilic effect. J Phys Chem B. 2008;112(51):16647-16653.

30. Qiao R, Roberts AP, Mount AS, Klaine SJ, Ke PC. Translocation of C60 and its derivatives across a lipid bilayer. Nano Lett. 2007;7(3): 614-619.

31. Lajud SA, Nagda DA, Qiao P, et al. A novel chitosan-hydrogel-based nanoparticle delivery system for local inner ear application. Otol Neurotol. 2015;36(2):341-347.

32. Luo S, Zhang E, Su Y, Cheng T, Shi C. A review of NIR dyes in cancer targeting and imaging. Biomaterials. 2011;32(29):7127-7138.

33. Roy S, Johnston AH, Newman TA. Cell-specific targeting in the mouse inner ear using nanoparticles conjugated with a neurotrophinderived peptide ligand: potential tool for drug delivery. Int J Pharm. 2010;390(2):214-224.

34. Swan EE, Mescher MJ, Sewell WF, Tao SL, Borenstein JT. Inner ear drug delivery for auditory applications. Adv Drug Deliv Rev. 2008;60(15): 1583-1599.

35. Akash MS, Rehman K. Recent progress in biomedical applications of pluronic (PF127): pharmaceutical perspectives. J Control Release. 2015;209:120-138.

36. Wang M, Lu P, Wu B, Tucker JD, Cloer C, Lu Q. High efficiency and low toxicity of polyethyleneimine modified Pluronics (PEI-Pluronic) as gene delivery carriers in cell culture and dystrophic mdx mice. J Mater Chem. 2012;22(13):6038-6046.

37. Kabanov AV, Batrakova EV, Alakhov VY. Pluronic ${ }^{\circledR}$ block copolymers as novel polymer therapeutics for drug and gene delivery. J Control Release. 2002;82(2):189-212.

38. Demina T, Grozdova I, Krylova O, et al. Relationship between the structure of amphiphilic copolymers and their ability to disturb lipid bilayers. Biochemistry. 2005;44(10):4042-4054.

39. Du XJ, Wang JL, Liu WW, et al. Regulating the surface poly(ethylene glycol) density of polymeric nanoparticles and evaluating its role in drug delivery in vivo. Biomaterials. 2015;69:1-11.

40. Roy S, Glueckert R, Johnston AH, et al. Strategies for drug delivery to the human inner ear by multifunctional nanoparticles. Nanomedicine (Lond). 2012;7(1):55-63.
International Journal of Nanomedicine

\section{Publish your work in this journal}

The International Journal of Nanomedicine is an international, peerreviewed journal focusing on the application of nanotechnology in diagnostics, therapeutics, and drug delivery systems throughout the biomedical field. This journal is indexed on PubMed Central,

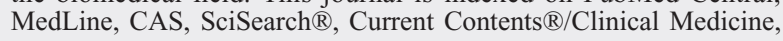

\section{Dovepress}

Journal Citation Reports/Science Edition, EMBase, Scopus and the Elsevier Bibliographic databases. The manuscript management system is completely online and includes a very quick and fair peer-review system, which is all easy to use. Visit http://www.dovepress.com/ testimonials.php to read real quotes from published authors. 